\title{
Applications of Fixed Point Theorems in the Theory of Generalized IFS
}

\author{
Alexandru Mihail and Radu Miculescu \\ Department of Mathematics, Bucharest University, Bucharest, Academiei Street 14, \\ 010014 Bucharest, Romania
}

Correspondence should be addressed to Radu Miculescu, miculesc@yahoo.com

Received 9 February 2008; Accepted 22 May 2008

Recommended by Hichem Ben-El-Mechaiekh

We introduce the notion of a generalized iterated function system (GIFS), which is a finite family of functions $f_{k}: X^{m} \rightarrow X$, where $(X, d)$ is a metric space and $m \in \mathbb{N}$. In case that $(X, d)$ is a compact metric space and the functions $f_{k}$ are contractions, using some fixed point theorems for contractions from $X^{m}$ to $X$, we prove the existence of the attractor of such a GIFS and its continuous dependence in the $f_{k}$ 's.

Copyright ( 2008 A. Mihail and R. Miculescu. This is an open access article distributed under the Creative Commons Attribution License, which permits unrestricted use, distribution, and reproduction in any medium, provided the original work is properly cited.

\section{Introduction}

We start with a short presentation of the notion of an iterated function system (IFS), one of the most common and most general ways to generate fractals. This will serve as a framework for our generalization of an iterated function system.

Then, we introduce the notion of a GIFS, which is a finite family of functions $f_{k}: X^{m} \rightarrow X$, where $(X, d)$ is a metric space and $m \in \mathbb{N}$. In case that $(X, d)$ is a compact metric space and the functions $f_{k}$ are contractions, using some fixed point theorems for contractions from $X^{m}$ to $X$, we prove the existence of the attractor of such a GIFS and its continuous dependence in the $f_{k}^{\prime}$ s.

IFSs were introduced in their present form by Hutchinson (see [1]) and popularized by Barnsley (see [2]). In the last period, IFSs have attracted much attention being used from researchers who work on autoregressive time series, engineer sciences, physics, and so forth. For applications of IFSs in image processing theory, in the theory of stochastic growth models, and in the theory of random dynamical systems, one can consult [3-5].

There is a current effort to extend Hutchinson's classical framework for fractals to more general spaces and infinite IFSs. 
Let us mention some papers containing results on this direction.

Results concerning infinite iterated function systems have been obtained for the case when the attractor is compact (see, e.g., [6] where the case of a countable iterated function system on a compact metric space is considered). In [7], we provide a general framework where attractors are nonempty closed and bounded subsets of topologically complete metric spaces and where the IFSs may be infinite, in contrast with the classical theory (see [2]), where only attractors that are compact metric spaces and IFSs that are finite are considered.

Gwóźdź-Łukawska and Jachymski [8] discuss the Hutchinson-Barnsley theory for infinite iterated function systems.

Łoziński et al. [9] introduce the notion of quantum iterated function systems (QIFSs) which is designed to describe certain problems of nonunitary quantum dynamics.

Käenmäki [10] constructs a thermodynamical formalism for very general iterated function systems.

Leśniak [11] presents a multivalued approach of infinite iterated function systems.

\section{Preliminaries}

Notations. Let $\left(X, d_{X}\right)$ and $\left(Y, d_{Y}\right)$ be two metric spaces.

As usual, $C(X, Y)$ denotes the set of continuous functions from $X$ to $Y$, and $\bar{d}: C(X, Y) \times$ $C(X, Y) \rightarrow \overline{\mathbb{R}}_{+}=\mathbb{R}_{+} \cup\{\infty\}$, defined by

$$
\bar{d}(f, g)=\sup _{x \in X} d_{Y}(f(x), g(x)),
$$

is the generalized metric on $C(X, Y)$.

For a sequence $\left(f_{n}\right)_{n}$ of elements of $C(X, Y)$ and $f \in C(X, Y), f_{n} \stackrel{s}{\rightarrow} f$ denotes the

pointwise convergence, $f_{n} \stackrel{u \cdot c}{\longrightarrow} f$ denotes the uniform convergence on compact sets, and $f_{n} \stackrel{u}{\rightarrow} f$ denotes the uniform convergence, that is, the convergence in the generalized metric $\bar{d}$.

Definition 2.1. Let $(X, d)$ be a complete metric space and let $m \in \mathbb{N}$. For a function $f: X^{m}=$ $\times_{k=1}^{m} X \rightarrow X$, the number

$$
\begin{aligned}
\inf \{c & : d\left(f\left(x_{1}, \ldots, x_{m}\right), f\left(y_{1}, \ldots, y_{m}\right)\right) \\
& \left.\leq c \max \left\{d\left(x_{1}, y_{1}\right), \ldots, d\left(x_{m}, y_{m}\right)\right\}, \forall x_{1}, \ldots, x_{m}, y_{1}, \ldots, y_{m} \in X\right\}
\end{aligned}
$$

which is the same as

$$
\sup \left\{d\left(f\left(x_{1}, \ldots, x_{m}\right), f\left(y_{1}, \ldots, y_{m}\right)\right): \max \left(d\left(x_{1}, y_{1}\right), \ldots, d\left(x_{m}, y_{m}\right)\right)\right\},
$$

where the sup is taken over $x_{1}, \ldots, x_{m}, y_{1}, \ldots, y_{m} \in X$ such that

$$
\max \left\{d\left(x_{1}, y_{1}\right), \ldots, d\left(x_{m}, y_{m}\right)\right\}>0,
$$

is denoted by $\operatorname{Lip}(f)$ and is called the Lipschitz constant of $f$.

A function $f: X^{m} \rightarrow X$ is called a Lipschitz function if $\operatorname{Lip}(f)<\infty$ and a Lipschitz contraction if $\operatorname{Lip}(f)<1$.

A function $f: X^{m} \rightarrow X$ is said to be a contraction if

$$
d\left(f\left(x_{1}, \ldots, x_{m}\right), f\left(y_{1}, \ldots, y_{m}\right)\right)<\max \left\{d\left(x_{1}, y_{1}\right), \ldots, d\left(x_{m}, y_{m}\right)\right\},
$$

for every $x_{1}, x_{2}, \ldots, x_{m}, y_{1}, y_{2}, \ldots, y_{m} \in X$, such that $x_{i} \neq y_{i}$ for some $i \in\{1,2, \ldots, n\}$. 
$L_{\text {Con }}(X)$ denotes the set

$$
\left\{f: X^{m} \longrightarrow X: \operatorname{Lip}(f)<1\right\}
$$

and $\operatorname{Con}_{m}(X)$ denotes the set

$$
\left\{f: X^{m} \longrightarrow X: f \text { is a contraction }\right\} .
$$

Remark 2.2. It is obvious that

$$
\operatorname{LCon}_{m}(X) \subseteq \operatorname{Con}_{m}(X)
$$

Notations. $P(X)$ denotes the family of all subsets of a given set $X$ and $D^{*}(X)$ denotes the set $P(X) \backslash\{\varnothing\}$.

For a subset $A$ of $D(X)$, by $A^{*}$ we mean $A \backslash\{\varnothing\}$.

Given a metric space $(X, d), \mathcal{K}(X)$ denotes the set of compact subsets of $X$ and $B(X)$ denotes the set of closed bounded subsets of $X$.

Remark 2.3. It is obvious that

$$
\mathcal{K}(X) \subseteq B(X) \subseteq D(X)
$$

Definition 2.4. For a metric space $(X, d)$, one considers on $p^{*}(X)$ the generalized HausdorffPompeiu pseudometric $h: D^{*}(X) \times D^{*}(X) \rightarrow[0,+\infty]$ defined by

$$
\begin{aligned}
h(A, B) & =\max (d(A, B), d(B, A)) \\
& =\inf \{r \in[0, \infty]: A \subseteq B(B, r), B \subseteq B(A, r)\},
\end{aligned}
$$

where

$$
\begin{aligned}
& B(A, r)=\{x \in X: d(x, A)<r\} \\
& d(A, B)=\sup _{x \in A} d(x, B)=\sup _{x \in A}\left(\inf _{y \in B} d(x, y)\right) .
\end{aligned}
$$

Remark 2.5. The Hausdorff-Pompeiu pseudometric is a metric on $\mathbb{B}^{*}(X)$ and, in particular, on $\mathcal{K}^{*}(X)$.

Remark 2.6. The metric spaces $\left(\mathbb{B}^{*}(X), h\right)$ and $\left(\mathcal{K}^{*}(X), h\right)$ are complete, provided that $(X, d)$ is a complete metric space (see $[2,7,12])$. Moreover, $\left(\mathcal{K}^{*}(X), h\right)$ is compact, provided that $(X, d)$ is a compact metric space (see [2]).

The following proposition gives the important properties of the Hausdorff-Pompeiu pseudometric (see $[2,13]$ ).

Proposition 2.7. Let $\left(X, d_{X}\right)$ and $\left(Y, d_{Y}\right)$ be two metric spaces. Then

(i) if $H$ and $K$ are two nonempty subsets of $X$, then

$$
h(H, K)=h(\bar{H}, \bar{K})
$$


(ii) if $\left(H_{i}\right)_{i \in I}$ and $\left(K_{i}\right)_{i \in I}$ are two families of nonempty subsets of $X$, then

$$
h\left(\bigcup_{i \in I} H_{i}, \bigcup_{i \in I} K_{i}\right) \leq \sup _{i \in I} h\left(H_{i}, K_{i}\right)
$$

(iii) if $H$ and $K$ are two nonempty subsets of $X$ and $f: X \rightarrow X$ is a Lipschitz function, then

$$
h(f(K), f(H)) \leq \operatorname{Lip}(f) h(K, H) .
$$

Definition 2.8. Let $(X, d)$ be a complete metric space and let $m \in \mathbb{N}$. A generalized iterated function system (in short a GIFS) on $X$ of order $m$, denoted by $\mathcal{S}=\left(X,\left(f_{k}\right)_{k=\overline{1, n}}\right)$, consists of a finite family of functions $\left(f_{k}\right)_{k=\overline{1, n}}, f_{k}: X^{m} \rightarrow X$ such that $f_{1}, \ldots, f_{n} \in \operatorname{Con}_{m}(X)$.

Definition 2.9. Let $f: X^{m} \rightarrow X$ be a continuous function. The function $F_{f}: \mathcal{K}^{*}(X)^{m} \rightarrow \mathcal{K}^{*}(X)$ defined by

$$
\begin{aligned}
F_{f}\left(K_{1}, K_{2}, \ldots, K_{m}\right) & =f\left(K_{1} \times K_{2} \times \cdots \times K_{m}\right) \\
& =\left\{f\left(x_{1}, x_{2}, \ldots, x_{m}\right): x_{j} \in K_{j}, \forall j \in\{1, \ldots, m\}\right\}
\end{aligned}
$$

is called the set function associated to the function $f$.

Definition 2.10. Given $\mathcal{S}=\left(X,\left(f_{k}\right)_{k=1, n}\right)$ a generalized iterated function system on $X$ of order $m$, the function $F_{\mathcal{S}}: \mathcal{K}^{*}(X)^{m} \rightarrow \mathcal{K}^{*}(X)$ defined by

$$
F_{\mathcal{S}}\left(K_{1}, K_{2}, \ldots, K_{m}\right)=\bigcup_{k=1}^{n} F_{f_{k}}\left(K_{1}, K_{2}, \ldots, K_{m}\right)
$$

is called the set function associated to $\mathcal{S}$.

Lemma 2.11. For a sequence $\left(f_{n}\right)_{n}$ of elements of $C\left(X^{m}, X\right)$ and $f \in C\left(X^{m}, X\right)$ such that $f_{n} \stackrel{u}{\rightarrow} f$ and for $K_{1}, K_{2}, \ldots, K_{m} \in \mathcal{K}^{*}(X)$, one has

$$
f_{n}\left(K_{1} \times K_{2} \times \cdots \times K_{m}\right) \longrightarrow f\left(K_{1} \times K_{2} \times \cdots \times K_{m}\right)
$$

in $\left(\mathcal{K}^{*}(X), h\right)$.

Proof. Indeed, the conclusion follows from the below inequality:

$$
\begin{aligned}
& h\left(f_{n}\left(K_{1} \times \cdots \times K_{m}\right), f\left(K_{1} \times \cdots \times K_{m}\right)\right) \\
& \quad \leq \sup _{x_{1} \in K_{1}, \ldots, x_{m} \in K_{m}} d\left(f_{n}\left(x_{1}, \ldots, x_{m}\right), f\left(x_{1}, \ldots, x_{m}\right)\right),
\end{aligned}
$$

which is valid for all $n \in \mathbb{N}$.

Proposition 2.12. Let $\left(X, d_{X}\right)$ and $\left(Y, d_{Y}\right)$ be two metric spaces and let $f_{n}, f \in C(X, Y)$ be such that $\sup _{n \geq 1} \operatorname{Lip}\left(f_{n}\right)<+\infty$ and $f_{n} \stackrel{s}{\rightarrow} f$ on a dense set in $X$.

Then

$$
\operatorname{Lip}(f) \leq \sup _{n \geq 1} \operatorname{Lip}\left(f_{n}\right), \quad f_{n} \stackrel{u \cdot c}{\longrightarrow} f .
$$


Proof. Set $M:=\sup _{n \geq 1} \operatorname{Lip}\left(f_{n}\right)$.

Let us consider $A=\left\{x \in X \mid f_{m}(x) \rightarrow f(x)\right\}$, which is a dense set in $X$, let $K$ be a compact set in $X$, and let $\varepsilon>0$.

Since $f$ is uniformly continuous on $K$, there exists $\delta \in(0, \varepsilon / 3(M+1))$ such that if $x, y \in K$ and $d_{X}(x, y)<\delta$, then

$$
d_{Y}(f(x), f(y))<\frac{\varepsilon}{3}
$$

Since $K$ is compact, there exist $x_{1}, x_{2}, \ldots, x_{n} \in K$ such that

$$
K \subseteq \bigcup_{i=1}^{n} B\left(x_{i}, \frac{\delta}{2}\right) \text {. }
$$

Taking into account the fact that $A$ is dense in $X$, we can choose $y_{1}, y_{2}, \ldots, y_{n} \in A$ such that $y_{1} \in B\left(x_{1}, \delta / 2\right), \ldots, y_{n} \in B\left(x_{n}, \delta / 2\right)$.

Since, for all $i \in\{1, \ldots, n\}, \lim _{m \rightarrow \infty} f_{m}\left(y_{i}\right)=f\left(y_{i}\right)$, there exists $m_{\varepsilon} \in \mathbb{N}$ such that for every $m \in \mathbb{N}, m \geq m_{\varepsilon}$, we have

$$
d_{Y}\left(f_{m}\left(y_{i}\right), f\left(y_{i}\right)\right)<\frac{\varepsilon}{3}
$$

for every $i \in\{1, \ldots, n\}$.

For $x \in K$, there exists $i \in\{1, \ldots, n\}$, such that $x \in B\left(x_{i}, \delta / 2\right)$ and therefore

$$
d_{X}\left(x, y_{i}\right) \leq d_{X}\left(x, x_{i}\right)+d_{X}\left(x_{i}, y_{i}\right)<\frac{\delta}{2}+\frac{\delta}{2}<\delta
$$

so

$$
d_{Y}\left(f\left(y_{i}\right), f(x)\right)<\frac{\varepsilon}{3}
$$

Hence, for $m \geq m_{\varepsilon}$, we have

$$
\begin{aligned}
d_{Y}\left(f_{m}(x), f(x)\right) & \leq d_{Y}\left(f_{m}(x), f_{m}\left(y_{i}\right)\right)+d_{Y}\left(f_{m}\left(y_{i}\right), f\left(y_{i}\right)\right)+d_{Y}\left(f\left(y_{i}\right), f(x)\right) \\
& \leq M d_{X}\left(x, y_{i}\right)+\frac{\varepsilon}{3}+\frac{\varepsilon}{3} \\
& \leq M \frac{\varepsilon}{3(M+1)}+\frac{2 \varepsilon}{3}<\varepsilon .
\end{aligned}
$$

Consequently, as $x$ was arbitrarily chosen in $K$, we infer that $f_{n} \stackrel{u}{\rightarrow} f$ on $K$, so

$$
f_{n} \stackrel{u \cdot c}{\longrightarrow} f .
$$

The inequality $\operatorname{Lip}(f) \leq \sup _{n \geq 1} \operatorname{Lip}\left(f_{n}\right)$ is obvious. ing lemma.

From Lemma 2.11 and Proposition 2.12, using Proposition 2.7(ii) we obtain the follow-

Lemma 2.13. Let $\left(X, d_{X}\right)$ be a complete metric space, let $m \in \mathbb{N}$, let $\mathcal{S}_{j}=\left(X,\left(f_{k}^{j}\right)_{k=\overline{1, n}}\right)$, where $j \in \mathbb{N}^{*}$, and let $\mathcal{S}=\left(X,\left(f_{k}\right)_{k=\overline{1, n}}\right)$ be generalized iterated function systems of order $m$, such that, for all $k \in$ $\{1, \ldots, n\}, f_{k}^{j} \stackrel{s}{\rightarrow} f_{k}$ on a dense subset of $X^{m}$

Then, for every $K_{1}, K_{2}, \ldots, K_{m} \in \mathcal{K}^{*}(X)$,

$$
F_{\mathcal{S}_{j}}\left(K_{1}, K_{2}, \ldots, K_{m}\right) \longrightarrow F_{\mathcal{S}}\left(K_{1}, K_{2}, \ldots, K_{m}\right),
$$

in $\left(\mathcal{K}^{*}(X), h\right)$. 


\section{The existence of the attractor of a GIFs for contractions}

In this section, $m$ is a natural number, $(X, d)$ is a compact metric space, and $\mathcal{S}=\left(X,\left(f_{k}\right)_{k=1, n}\right)$ is a generalized iterated function system on $X$ of order $m$.

First, we prove that $F_{\mathcal{S}}: \mathcal{K}^{*}(X)^{m} \rightarrow \mathcal{K}^{*}(X)$ is a contraction (Proposition 3.1), then, using some results concerning the fixed points of contractions from $X^{m}$ to $X$ (Theorem 3.4), we prove the existence of the attractor of $\mathcal{S}$ (Theorem 3.5) and its continuous dependence in the $f_{k}$ 's (Theorem 3.7).

The following proposition is crucial.

Proposition 3.1. $F_{\mathcal{S}}: \mathcal{K}^{*}(X)^{m} \rightarrow \mathcal{K}^{*}(X)$ is a contraction.

Proof. By Proposition 2.7, we have

$$
\begin{aligned}
& h\left(F_{\mathcal{S}}\left(K_{1}, K_{2}, \ldots, K_{m}\right), F_{\mathcal{S}}\left(H_{1}, H_{2}, \ldots, H_{m}\right)\right) \\
& \quad=h\left(\bigcup_{k=1}^{n} f_{k}\left(K_{1} \times K_{2} \times \cdots \times K_{m}\right), \bigcup_{k=1}^{n} f_{k}\left(H_{1} \times H_{2} \times \cdots \times H_{m}\right)\right) \\
& \quad=h\left(\bigcup_{k=1}^{n} F_{f_{k}}\left(K_{1}, K_{2}, \ldots, K_{m}\right), \bigcup_{k=1}^{n} F_{f_{k}}\left(H_{1}, H_{2}, \ldots, H_{m}\right)\right) \\
& \quad \leq \max \left\{h\left(f_{1}\left(K_{1} \times \cdots \times K_{m}\right), f_{1}\left(H_{1} \times \cdots \times H_{m}\right)\right), \ldots, h\left(f_{n}\left(K_{1} \times \cdots \times K_{m}\right),\right.\right. \\
& \left.\left.\quad f_{n}\left(H_{1} \times \cdots \times H_{m}\right)\right)\right\} \\
& \leq \max \left\{h\left(H_{1}, K_{1}\right), \ldots, h\left(H_{m}, K_{m}\right)\right\},
\end{aligned}
$$

for all $K_{1}, \ldots, K_{m}, H_{1}, \ldots, H_{m} \in \mathcal{K}^{*}(X)$.

It remains to prove that the above inequality is strict.

Let $K_{1}, K_{2}, \ldots, K_{m}, H_{1}, H_{2}, \ldots, H_{m} \in \mathcal{K}^{*}(X)$ be fixed such that $K_{i} \neq H_{i}$ for some $i \in$ $\{1,2, \ldots, m\}$.

Since

$$
\begin{aligned}
& h\left(F_{\mathcal{S}}\left(K_{1}, \ldots, K_{m}\right), F_{\mathcal{S}}\left(H_{1}, \ldots, H_{m}\right)\right) \\
& \quad=\max \left(d\left(F_{\mathcal{S}}\left(K_{1}, \ldots, K_{m}\right), F_{\mathcal{S}}\left(H_{1}, \ldots, H_{m}\right)\right), d\left(F_{\mathcal{S}}\left(H_{1}, \ldots, H_{m}\right), F_{\mathcal{S}}\left(K_{1}, \ldots, K_{m}\right)\right)\right),
\end{aligned}
$$

we can suppose, by using symmetry arguments, that

$$
h\left(F_{\mathcal{S}}\left(K_{1}, \ldots, K_{m}\right), F_{\mathcal{S}}\left(H_{1}, \ldots, H_{m}\right)\right)=d\left(F_{\mathcal{S}}\left(K_{1}, \ldots, K_{m}\right), F_{\mathcal{S}}\left(H_{1}, \ldots, H_{m}\right)\right),
$$

that is,

$$
\begin{aligned}
& h\left(\bigcup_{k=1}^{n} f_{k}\left(K_{1} \times \cdots \times K_{m}\right), \bigcup_{k=1}^{n} f_{k}\left(H_{1} \times \cdots \times H_{m}\right)\right) \\
& \quad=d\left(\bigcup_{k=1}^{n} f_{k}\left(K_{1} \times \cdots \times K_{m}\right), \bigcup_{k=1}^{n} f_{k}\left(H_{1} \times \cdots \times H_{m}\right)\right) .
\end{aligned}
$$


Let us note that for every $K_{1}, K_{2}, \ldots, K_{m} \in \mathcal{K}^{*}(X)$, since $f_{1}, \ldots, f_{n}$ are continuous functions, $F_{S}\left(K_{1}, K_{2}, \ldots, K_{m}\right)=\bigcup_{k=1}^{n} f_{j}\left(K_{1}, K_{2}, \ldots, K_{m}\right)$ is a compact set.

Since for all $K_{1}, K_{2}, \ldots, K_{m}, H_{1}, H_{2}, \ldots, H_{m} \in \mathcal{K}^{*}(X)$, the product topological space $\{1,2, \ldots, n\} \times\left(\times_{j=1}^{m} K_{j}\right)$, where $\{1,2, \ldots, n\}$ is endowed with the discrete topology, is compact and the function $t:\{1,2, \ldots, n\} \times\left(\times_{j=1}^{m} K_{j}\right) \rightarrow \mathbb{R}$, given by

$$
t\left(k, x_{1}, x_{2}, \ldots, x_{m}\right)=d\left(f_{k}\left(x_{1}, x_{2}, \ldots, x_{m}\right), F_{\mathcal{S}}\left(H_{1}, H_{2}, \ldots, H_{m}\right)\right)
$$

is continuous and

$$
\begin{aligned}
d\left(F_{\mathcal{S}}\left(K_{1}, K_{2}, \ldots, K_{m}\right), F_{\mathcal{S}}\left(H_{1}, H_{2}, \ldots, H_{m}\right)\right) \\
\quad=d\left(\bigcup_{k=1}^{n} f_{j}\left(K_{1}, K_{2}, \ldots, K_{m}\right), F_{\mathcal{S}}\left(H_{1}, H_{2}, \ldots, H_{m}\right)\right) \\
=\sup _{\left(j, x_{1}, x_{2}, \ldots, x_{m}\right) \in\{1,2, \ldots, n\} \times\left(\times_{j=1}^{m} K_{j}\right)}\left\{d\left(f_{j}\left(x_{1}, x_{2}, \ldots, x_{m}\right), F_{\mathcal{S}}\left(H_{1}, H_{2}, \ldots, H_{m}\right)\right)\right\} \\
\left.=\sup _{\left(j, x_{1}, x_{2}, \ldots, x_{m}\right) \in\{1,2, \ldots, n\} \times\left(\times_{j=1}^{m} K_{j}\right)}\left\{t\left(k, x_{1}, x_{2}, \ldots, x_{m}\right), F_{\mathcal{S}}\left(H_{1}, H_{2}, \ldots, H_{m}\right)\right)\right\},
\end{aligned}
$$

it follows that there exist $\bar{k} \in\{1,2, \ldots, n\}, \bar{x}_{1} \in K_{1}, \bar{x}_{2} \in K_{2}, \ldots$, and $\bar{x}_{m} \in K_{m}$ such that

$$
\begin{aligned}
d\left(f_{\bar{k}}\left(\bar{x}_{1}, \ldots, \bar{x}_{m}\right), F_{\mathcal{S}}\left(H_{1}, \ldots, H_{m}\right)\right) & =d\left(F_{\mathcal{S}}\left(K_{1}, \ldots, K_{m}\right), F_{\mathcal{S}}\left(H_{1}, \ldots, H_{m}\right)\right) \\
& =h\left(F_{\mathcal{S}}\left(K_{1}, \ldots, K_{m}\right), F_{\mathcal{S}}\left(H_{1}, \ldots, H_{m}\right)\right) .
\end{aligned}
$$

Let us also note that since for all $k \in\{1, \ldots, n\}$, the function $t_{k}: H_{k} \rightarrow \mathbb{R}$, given by

$$
t_{k}(y)=d\left(\bar{x}_{k}, y\right)
$$

is continuous, $H_{k}$ is a compact set, and $d\left(\bar{x}_{k}, H_{k}\right)=\inf \left\{d\left(\bar{x}_{k}, y\right): y \in H_{k}\right\}$, it follows that there exists $\bar{y}_{k} \in H_{k}$ such that

$$
d\left(\bar{x}_{k}, \bar{y}_{k}\right)=d\left(\bar{x}_{k}, H_{k}\right)
$$

thus

$$
d\left(\bar{x}_{k}, \bar{y}_{k}\right)=d\left(\bar{x}_{k}, H_{k}\right) \leq d\left(K_{k}, H_{k}\right) \leq h\left(K_{k}, H_{k}\right)
$$

Now we are able to prove that

$$
h\left(F_{\mathcal{S}}\left(K_{1}, K_{2}, \ldots, K_{m}\right), F_{\mathcal{S}}\left(H_{1}, H_{2}, \ldots, H_{m}\right)\right)<\max \left\{h\left(H_{1}, K_{1}\right), \ldots, h\left(H_{m}, K_{m}\right)\right\}
$$

for all $K_{1}, K_{2}, \ldots, K_{m}, H_{1}, H_{2}, \ldots, H_{m} \in \mathcal{K}^{*}(X)$ such that $K_{i} \neq H_{i}$ for some $i \in\{1,2, \ldots, m\}$. 
Indeed, we have

$$
\begin{aligned}
h\left(F_{\mathcal{S}}\right. & \left.\left(K_{1}, K_{2}, \ldots, K_{m}\right), F_{\mathcal{S}}\left(H_{1}, H_{2}, \ldots, H_{m}\right)\right) \\
& =d\left(f_{\bar{k}}\left(\bar{x}_{1}, \bar{x}_{2}, \ldots, \bar{x}_{m}\right), F_{\mathcal{S}}\left(H_{1}, H_{2}, \ldots, H_{m}\right)\right) \\
& =d\left(f_{\bar{k}}\left(\bar{x}_{1}, \bar{x}_{2}, \ldots, \bar{x}_{m}\right), \bigcup_{k=1}^{n} f_{k}\left(H_{1} \times H_{2} \times \cdots \times H_{m}\right)\right) \\
& =\inf \left\{d\left(f_{\bar{k}}\left(\bar{x}_{1}, \ldots, \bar{x}_{m}\right), f_{k}\left(y_{1}, \ldots, y_{m}\right)\right): k \in\{1,2, \ldots, n\}, y_{1} \in H_{1}, \ldots, y_{m} \in H_{m}\right\} \\
& \leq d\left(f_{\bar{k}}\left(\bar{x}_{1}, \ldots, \bar{x}_{m}\right), f_{\bar{k}}\left(\bar{y}_{1}, \ldots, \bar{y}_{m}\right)\right) .
\end{aligned}
$$$$
\text { If } \bar{x}_{k}=\bar{y}_{k} \text {, for all } k \in\{1,2, \ldots, n\} \text {, then }
$$

$$
h\left(F_{\mathcal{S}}\left(K_{1}, K_{2}, \ldots, K_{m}\right), F_{\mathcal{S}}\left(H_{1}, H_{2}, \ldots, H_{m}\right)\right)=0,
$$

so the above claim is true.

Otherwise, we have

$$
\begin{aligned}
h\left(F_{\mathcal{S}}\left(K_{1}, K_{2}, \ldots, K_{m}\right), F_{\mathcal{S}}\left(H_{1}, H_{2}, \ldots, H_{m}\right)\right) & \leq d\left(f_{\bar{k}}\left(\bar{x}_{1}, \ldots, \bar{x}_{m}\right), f_{\bar{k}}\left(\bar{y}_{1}, \ldots, \bar{y}_{m}\right)\right) \\
& <\max \left\{d\left(\bar{x}_{1}, \bar{y}_{k}\right), \ldots, d\left(\bar{x}_{m}, \bar{y}_{m}\right)\right\} \\
& =\max \left\{d\left(\bar{x}_{1}, H_{1}\right), \ldots, d\left(\bar{x}_{m}, H_{m}\right)\right\} \\
& \leq \max \left\{d\left(K_{1}, H_{1}\right), \ldots, d\left(K_{m}, H_{m}\right)\right\} \\
& \leq \max \left\{h\left(K_{1}, H_{1}\right), \ldots, h\left(K_{m}, H_{m}\right)\right\},
\end{aligned}
$$

for all $K_{1}, K_{2}, \ldots, K_{m}, H_{1}, H_{2}, \ldots, H_{m} \in \mathcal{K}^{*}(X)$ such that $K_{i} \neq H_{i}$ for some $i \in\{1,2, \ldots, m\}$.

Let us recall the following result.

Theorem 3.2. For a contraction $f: X \rightarrow X$, there exists a unique $\alpha \in X$ such that $f(\alpha)=\alpha$.

For every $x_{0} \in X$, the sequence $\left(x_{k}\right)_{k \geq 0}$, defined by

$$
x_{k+1}=f\left(x_{k}\right),
$$

for all $k \in \mathbb{N}$, is convergent to $\alpha$.

Moreover, if $f_{j}: X \rightarrow X$, where $j \in \mathbb{N}$, are contractions having the fixed points $\alpha_{j}$, such that $f_{j} \stackrel{s}{\rightarrow} f$ on a dense subset of $X$, then

$$
\lim _{j \rightarrow \infty} \alpha_{j}=\alpha
$$

Let us mention that the first part of Theorem 3.2 is due to Edelstein (see [14]).

Theorem 3.3. Let $f: X \rightarrow X$ be a function having the property that there exists $p \in \mathbb{N}^{*}$ such that $f^{[p]}$ is a contraction.

Then there exists a unique $\alpha \in X$ such that $f(\alpha)=\alpha$ and, for any $x_{0} \in X$, the sequence $\left(x_{k}\right)_{k \geq 0}$ defined by $x_{k+1}=f\left(x_{k}\right)$ is convergent to $\alpha$. 
Proof. It is clear that $f^{[p]}$ has a unique fixed point $\alpha \in X$ and, for every $y_{0} \in X$, the sequence $\left(y_{k}\right)_{k \geq 1}$ defined by $y_{k+1}=f^{[p]}\left(y_{k}\right)$ is convergent to $\alpha$.

In particular for $y_{0}^{j}=f^{[j]}\left(x_{0}\right)$, where $x_{0} \in X$ and $j \in\{0,1, \ldots, p-1\}$, the sequence $\left(y_{n}^{j}=f^{[n p+j]}\left(x_{0}\right)\right)_{n \geq 0}$ is convergent to $\alpha$.

It follows that the sequence $\left(x_{k}\right)_{k \geq 0}$, defined by $x_{k+1}=f\left(x_{k}\right)$, is convergent to $\alpha$.

Since every fixed point of $f$ is a fixed point of $f^{[p]}$, it follows that $\alpha$ is the unique fixed point of $f$.

Theorem 3.4. Given a contraction $f: X^{m} \rightarrow X$, there exists a unique $\alpha \in X$ such that

$$
f(\alpha, \alpha, \ldots, \alpha)=\alpha
$$

For every $x_{0}, x_{1}, \ldots, x_{m-1} \in X$, the sequence $\left(x_{k}\right)_{k \geq 0}$ defined by

$$
x_{k+m}=f\left(x_{k+m-1}, x_{k+m-2}, \ldots, x_{k}\right),
$$

for all $k \in \mathbb{N}$, is convergent to $\alpha$.

Moreover, if for every $j \in \mathbb{N}, f_{j}: X^{m} \rightarrow X$ is a contraction and $\alpha_{j}$ is the unique point of $X$ having the property that

$$
f_{j}\left(\alpha_{j}, \alpha_{j}, \ldots, \alpha_{j}\right)=\alpha_{j}
$$

then

$$
\lim _{j \rightarrow \infty} \alpha_{j}=\alpha
$$

provided that $f_{j} \stackrel{s}{\rightarrow} f$ on a dense subset of $X^{m}$.

Proof. Let $g: X \rightarrow X$ and $g_{j}: X \rightarrow X$ be the functions defined by

$$
\begin{aligned}
g(x) & =f(x, x, \ldots, x), \\
g_{j}(x) & =f_{j}(x, x, \ldots, x),
\end{aligned}
$$

for every $x \in X$.

Then $g$ and $g_{j}$ are contractions.

It follows, using Theorem 3.2, that there exist unique $\alpha \in X$ and $\alpha_{j} \in X$ such that

$$
\begin{gathered}
\alpha=g(\alpha)=f(\alpha, \alpha, \ldots, \alpha), \\
\alpha_{j}=g\left(\alpha_{j}\right)=f\left(\alpha_{j}, \alpha_{j}, \ldots, \alpha_{j}\right), \\
\lim _{j \rightarrow \infty} \alpha_{j}=\alpha .
\end{gathered}
$$


The function $h: X^{m} \rightarrow X^{m}$, given by

$$
\begin{aligned}
h\left(x_{0}, x_{1}, \ldots, x_{m-1}\right) & =\left(x_{1}, x_{2}, \ldots, x_{m-1}, f\left(x_{0}, x_{1}, \ldots, x_{m-1}\right)\right) \\
& =\left(x_{1}, x_{2}, \ldots, x_{m-1}, x_{m}\right)
\end{aligned}
$$

for all $x_{0}, x_{1}, \ldots, x_{m-1} \in X$, fulfills the conditions of Theorem 3.3 (taking $p=m$ ).

Therefore, there exists $\left(\beta_{1}, \beta_{2}, \ldots, \beta_{m}\right) \in X^{m}$ such that

$$
h\left(\beta_{1}, \beta_{2}, \ldots, \beta_{m}\right)=\left(\beta_{1}, \beta_{2}, \ldots, \beta_{m}\right),
$$

so

$$
\beta_{1}=\beta_{2}=\cdots=\beta_{m}=f\left(\beta_{1}, \beta_{2}, \ldots, \beta_{m}\right) .
$$

Hence,

$$
\beta_{1}=\beta_{2}=\cdots=\beta_{m}=\alpha \text {. }
$$

Then,

$$
\begin{aligned}
\lim _{l \rightarrow \infty} h^{[l]}\left(x_{0}, x_{1}, \ldots, x_{m-1}\right) & =\lim _{l \rightarrow \infty}\left(x_{l}, x_{l+1}, \ldots, x_{l+m-1}\right) \\
& =(\alpha, \alpha, \ldots, \alpha),
\end{aligned}
$$

so we conclude our claim.

Using Proposition 3.1, Theorem 3.4, and Lemma 2.13, we obtain the following two results.

Theorem 3.5. Given a generalized iterated function system of order $m \mathcal{S}=\left(X,\left(f_{k}\right)_{k=\overline{1, n}}\right)$, there exists a unique $A(\mathcal{S}) \in \mathcal{K}^{*}(X)$ such that

$$
F_{\mathcal{S}}(A(\mathcal{S}), A(\mathcal{S}), \ldots, A(\mathcal{S}))=A(\mathcal{S})
$$

Moreover, for any $H_{0}, H_{1}, \ldots, H_{m-1} \in \mathcal{K}^{*}(X)$, the sequence $\left(H_{n}\right)_{n \geq 0}$, defined by

$$
H_{n+m}=F_{S}\left(H_{n+m-1}, H_{n+m-2}, \ldots, H_{n}\right),
$$

for all $n \in \mathbb{N}$, is convergent to $A(\mathcal{S})$.

Definition 3.6. Let $m$ be a fixed natural number, let $(X, d)$ be a compact metric space, and let $\mathcal{S}=\left(X,\left(f_{k}\right)_{k=\overline{1, n}}\right)$ be a generalized iterated function system on $X$ of order $m$.

The unique set $A(\mathcal{S})$ given by the previous theorem is called the attractor of the GIFS $S$.

Theorem 3.7. If $\mathcal{S}=\left(X,\left(f_{k}\right)_{k=\overline{1, n}}\right)$ and $\mathcal{S}_{j}=\left(X,\left(f_{k}^{j}\right)_{k=\overline{1, n}}\right)$, where $j \in \mathbb{N}$, are GIFS of order $m$ such that, for every $k \in\{1,2, \ldots, n\}, f_{k}^{j} \stackrel{s}{\rightarrow} f_{k}$ on a dense set in $X^{m}$, then

$$
A\left(\mathcal{S}_{j}\right) \longrightarrow A(\mathcal{S})
$$




\section{Acknowledgments}

The authors want to thank the referees whose generous and valuable remarks and comments brought improvements to the paper and enhanced clarity. The work was supported by GAR $30 / 2007$.

\section{References}

[1] J. E. Hutchinson, "Fractals and self-similarity," Indiana University Mathematics Journal, vol. 30, no. 5, pp. 713-747, 1981.

[2] M. F. Barnsley, Fractals Everywhere, Academic Press Professional, Boston, Mass, USA, 2nd edition, 1993.

[3] J. H. Elton and M. Piccioni, "Iterated function systems arising from recursive estimation problems," Probability Theory and Related Fields, vol. 91, no. 1, pp. 103-114, 1992.

[4] B. Forte and E. R. Vrscay, "Solving the inverse problem for function/image approximation using iterated function systems-I: theoretical basis," Fractals, vol. 2, no. 3, pp. 325-334, 1994.

[5] L. Montrucchio and F. Privileggi, "Fractal steady states in stochastic optimal control models," Annals of Operations Research, vol. 88, pp. 183-197, 1999.

[6] N.-A. Secelean, "Countable iterated function systems," Far East Journal of Dynamical Systems, vol. 3, no. 2, pp. 149-167, 2001.

[7] R. Miculescu and A. Mihail, "Lipscomb's space $\omega^{A}$ is the attractor of an infinite IFS containing affine transformations of $l^{2}(A)$," Proceedings of the American Mathematical Society, vol. 136, no. 2, pp. 587-592, 2008.

[8] G. Gwóźdź-Łukawska and J. Jachymski, "The Hutchinson-Barnsley theory for infinite iterated function systems," Bulletin of the Australian Mathematical Society, vol. 72, no. 3, pp. 441-454, 2005.

[9] A. Łoziński, K. Życzkowski, and W. Słomczyński, "Quantum iterated function systems," Physical Review E, vol. 68, no. 4, Article ID 046110, 9 pages, 2003.

[10] A. Käenmäki, "On natural invariant measures on generalised iterated function systems," Annales Academiæ Scientiarium Fennicæ. Mathematica, vol. 29, no. 2, pp. 419-458, 2004.

[11] K. Leśniak, "Infinite iterated function systems: a multivalued approach," Bulletin of the Polish Academy of Sciences. Mathematics, vol. 52, no. 1, pp. 1-8, 2004.

[12] K. J. Falconer, The Geometry of Fractal Sets, vol. 85 of Cambridge Tracts in Mathematics, Cambridge University Press, Cambridge, UK, 1986.

[13] K. J. Falconer, Fractal Geometry: Mathematical Foundations and Applications, John Wiley \& Sons, Chichester, UK, 1990.

[14] M. Edelstein, "On fixed and periodic points under contractive mappings," Journal of the London Mathematical Society, vol. 37, pp. 74-79, 1962. 